\title{
Blockade of Very Late Antigen-4 Integrin Binding to Fibronectin with Connecting Segment-1 Peptide Reduces Accelerated Coronary Arteriopathy in Rabbit Cardiac Allografts
}

\author{
Silvana Molossi, “ Mariano Elices, ${ }^{\ddagger}$ Thomas Arthenius, ${ }^{\ddagger}$ Roberto Diaz, * Claire Coulber, * and Marlene Rabinovitch * \\ *Departments of Pediatrics, Pathology, and Medicine, University of Toronto and Division of Cardiovascular Research, The Hospital \\ For Sick Children, Toronto, Ontario M5G 1X8, Canada; and ${ }^{\ddagger}$ Cytel Corporation, San Diego, California 92121
}

\begin{abstract}
Graft arteriopathy, a leading cause of cardiac allograft failure, is associated with increased intimal smooth muscle cells, inflammatory cells, and accumulation of extracellular matrix. We hypothesized that cellular fibronectin plays a pivotal role in the progression of the allograft arteriopathy by directing the transendothelial traficking of inflammatory cells through interaction of the connecting segment-1 (CS1) motif with the very late antigen-4 (VLA-4) integrin, and tested this in vivo using a blocking peptide. Cholesterolfed rabbits underwent heterotopic cardiac transplantation without immunosuppression. The treatment group $(n=7)$ received a synthetic CS1 peptide (1 $\mathrm{mg} / \mathrm{kg}$ per d, subcutaneously), and the controls $(n=7)$ received an inactive peptide (1 $\mathrm{mg} / \mathrm{kg}$ per d, subcutaneously). At 7-8 d after transplantation, hearts were harvested and sectioned for morphometric analysis and immunohistochemical studies. We observed $a>50 \%$ decrease in the incidence $(P<0.001)$ and severity $(P<0.001)$ of donor coronary artery intimal thickening in the CS1-treated compared with the control group. These findings correlated with reduced infiltration of $T$ cells $(P<0.05)$, a trend toward decreased expression of adhesion molecules $(P<0.06)$, and less accumulation of fibronectin $(P<0.03)$. Our data suggest that the VLA-4-fibronectin interaction is critical to the progression of the allograft arteriopathy by perpetuating the immune-inflammatory response in the vessel wall. (J. Clin. Invest. 1995. 95:26012610.) Key words: vasculopathy • matrix • inflammation • vascular rejection • transplantation
\end{abstract}

\section{Introduction}

The results of cardiac transplantation have been improved considerably by the advent of better immunosuppression therapy, but long-term survival is often limited by rapidly developing coronary arteriopathy (1). The pathophysiology of this complication has been studied extensively and an immune-inflammatory response is believed to be a cause of progressive coronary

Address correspondence to Marlene Rabinovitch, M.D., Division of Cardiovascular Research, The Hospital For Sick Children, 555 University Avenue, Toronto, Ontario M5G 1X8, Canada. Phone: 416-8135918; FAX: 416-813-7480.

Received for publication 18 July 1994 and in revised form 23 December 1994.

J. Clin. Invest.

(c) The American Society for Clinical Investigation, Inc. $0021-9738 / 95 / 06 / 2601 / 10 \quad \$ 2.00$

Volume 95, June 1995, 2601-2610 artery neointimal thickening. The process involves the recruitment of inflammatory cells and release of soluble factors, i.e., cytokines and growth factors, leading to smooth muscle cell migration and proliferation and deposition of newly formed extracellular matrix (2-4). These soluble factors also modulate the functional status of circulating immune-reactive cells and the vascular endothelium, allowing interaction through the expression and activation of receptors and counterreceptors, i.e., intercellular adhesion molecule-1 (ICAM-1) ${ }^{1}$ and vascular cell adhesion molecule-1 (VCAM-1) $(5,6)$.

Various extracellular matrix components appear to have a determining role in lymphocyte trafficking (7) through their interaction with cell surface antigens, namely integrin receptors (8), and the latter, in turn, exert synergistic effects on T cell activation $(9,10)$ and cytokine release $(10)$. The potential of fibronectin, an extracellular matrix component, as a ligand for lymphocytes has been extensively investigated $(7,8,11-13)$. The presence of receptors on lymphocytes that bind fibronectin has suggested that this molecule plays a role in lymphocyte adhesion (11). The $\alpha 4 \beta 1$ (also called very late antigen- 4 [VLA-4]) and $\alpha 5 \beta 1$ (also called VLA-5) integrins, present on a variety of cells including lymphocytes, bind to specific sites on the fibronectin molecule, i.e., the connecting segment-1 (CS1) motif present in an alternatively spliced (V) region (8, 14 ) and the arginine-glycine-aspartate (RGD) sequence present in the cell adhesion domain (15-17), respectively. It has been shown that interactions between fibronectin and inflammatory cells, including eosinophils and monocytes as well as lymphocytes, enhance migration $(16,18-20)$. Fibronectin potentiates lymphocyte proliferation $(9,15)$ and also prolongs eosinophil survival in culture by triggering production of cytokines (21).

Takeuchi et al. (22) reported that increased expression of VLA-4 molecules in peripheral blood lymphocytes of systemic lupus erythematosus patients with vasculitis was associated with enhanced adhesion to the CS1 motif of fibronectin in vitro. Similar findings were published by Laff́on and colleagues (23) when they analyzed $T$ cells from the inflamed synovium of patients with rheumatoid arthritis. Since VLA-4 integrin receptors are upregulated on inflammatory cells, a useful therapeutic strategy may be to block VLA-4 interactions with its counterreceptors on endothelial cell surfaces or with fibronectin, by specific antibodies or synthetic peptides. In this regard, Elices et al. (24) have recently reported CS1-containing fibronectin isoforms on the synovial endothelium of rheumatoid arthritis patients and, also, that adhesion of $\mathrm{T}$ lymphoblastoid cells to this endothelium could be abrogated either by an anti- $\alpha 4$ integrin

1. Abbreviations used in this paper: CS1, connecting segment-1; ICAM1 , intercellular adhesion molecule-1; TNF- $\alpha$ sr, TNF- $\alpha$ soluble receptor; VCAM-1, vascular cell adhesion molecule-1; VLA, very late antigen. 
antibody or by the CS1 peptide. In addition, CS1 peptide was shown to decrease lymphocyte migration through high endothelial venule cells, reinforcing a role for fibronectin in the recruitment of these inflammatory cells (25).

We have demonstrated previously in vivo that an immuneinflammatory response in donor coronary arteries was associated with increased expression of both fibronectin and IL- $1 \beta$, using a piglet heterotopic cardiac transplant model of induced allograft arteriopathy (26). Further in vitro studies showed that donor coronary artery endothelial and smooth muscle cells produced increased amounts of fibronectin which was regulated by increased endogenous IL- $\beta(3,4)$ and TNF- $\alpha(27)$. The functional significance of this feature was pursued using a heterotopic cardiac transplant model in cholesterol-fed rabbits in which anticytokine therapy, namely TNF- $\alpha$ soluble receptor (TNF- $\alpha$ sr), effectively decreased the incidence and severity of graft arteriopathy (28). Mechanistically, this was associated with decreased expression of fibronectin and a reduced immuneinflammatory response, including downregulation of ICAM-1 and VCAM-1 expression (29), in the treated animals compared with controls (28). Taken together these observations suggest that the increased subendothelial fibronectin, in addition to its potential role in directing smooth muscle cell migration (30), may be responsible for trafficking of immune-inflammatory cells through the vessel wall.

This study was, therefore, carried out to address in vivo, using an experimental rabbit cardiac transplant model, the role of fibronectin in mediating transendothelial lymphocyte trafficking and in influencing the development and severity of the accelerated graft arteriopathy. 1 wk after cardiac transplantation, we observed a $>50 \%$ reduction in the number and severity of allograft coronary artery lesions in a group of animals treated with CS1 peptide compared with a control group, where a scrambled (inactive) form of CS1 peptide was used. The decreased neointimal formation was associated with reduction in $\mathrm{T}$ cell infiltration, expression of cell adhesion molecules, and accumulation of fibronectin. Severe myocardial rejection, however, occurred despite CS1 treatment, suggesting qualitative or quantitative differences in immune mechanisms. Thus, our observations suggest that a novel adjunctive therapeutic approach, directed at blocking the interaction between integrins and fibronectin, may be useful either in preventing the development or limiting the severity of graft arteriopathy after cardiac transplantation.

\section{Methods}

\section{Experimental animal model}

New Zealand White female rabbits (Charles River Lab., Saint Laurent, Quebec, Canada) between 3.5 and $4 \mathrm{~kg}$ underwent heterotopic cardiac transplant following an experimental protocol previously described (28, 31 ) and approved by the Animal Care Committee of The Hospital For Sick Children, Toronto. The animals were unselected to favor an HLAmismatch, the host rabbits were Pasteurella-free, and the donors were outbred animals. Both host and donor rabbits were fed Purina 5321$0.5 \%$ cholesterol diet (Research Diets Inc., New Brunswick, NJ), a strategy which has proven useful in accelerating the process of allograft arteriopathy (31). The diet was commenced $4 \mathrm{~d}$ before the transplant and continued in the recipient of the transplant until the completion of the experimental period. The technique of heterotopic cardiac transplantation has been described previously (28). Briefly, a vertical incision was performed in the anterior aspect of the neck of the recipient rabbit and the left common carotid artery and the ipsilateral external jugular vein were isolated. The cardiac allograft was placed in the neck by anastomosing the aorta end-to-side to the recipient's carotid artery and the pulmonary artery end-to-side to the recipient's external jugular vein, after a total period of ischemia for the donor hearts of FF7E $30 \mathrm{~min}$. Postoperative care was in compliance with the Principles of Laboratory Animal Care formulated by the Canadian National Society for Medical Research.

The treatment consisted of a synthetic CS1 tetrapeptide (VLA-4 inhibitor), containing the active sequence present in the fibronectin molecule $(32,33)$, and a scrambled form of the same synthetic tetrapeptide, scrambled CS1 (control peptide), resulting in no inhibition of VLA-4. Both peptides were synthesized at Cytel Corporation (San Diego, CA). The exact sequence of the CS1 peptide (VLA-4 inhibitor) is phenylacetic acid-Leu-Asp-Phe-d-Pro-amide and of the scrambled CS1 (control) peptide is phenylacetic acid-Asp-Leu-Phe-d-Pro-amide. The $\mathrm{IC}_{50}$ (50\% inhibitory concentration) of CS1 scrambled peptide for in vitro inhibition of VLA-4 to CS1 is $0.2-0.5 \mu \mathrm{M}$ and that for inhibition of VLA-4 to VCAM-1 is $950 \mu \mathrm{M}$. It is known that the peptide CS1 is resistant to degradation by specific enzymes for at least $24 \mathrm{~h}$ at $37^{\circ} \mathrm{C}$. Beginning the day of the transplant, the animals were randomized and treated with either scrambled CS1 peptide (control group) $1 \mathrm{mg} / \mathrm{kg}$ subcutaneously (s.c.) or the active form of CS1 peptide $1 \mathrm{mg} / \mathrm{kg}$ s.c. The doses of the peptides were empirically extrapolated to the in vivo model based on preliminary in vitro studies performed at Cytel Corporation. While it is still not possible to obtain serum levels for either peptide, pharmacokinetic studies are currently being undertaken. No other immunosuppression therapy was administered. The grafts were monitored daily by palpation and maintained for 7-8 d, an end point we had described previously which was associated with myocardial rejection (impaired cardiac contractility) and development of the allograft arteriopathy in this model (28). 14 animals in total were studied in the control $(n=7)$ and CS1-treated $(n=7)$ groups.

Preparation of the hearts. The animals were killed using a lethal dose of euthanol (480 mg intravenously) (MTC Pharmaceutical, Cambridge, Ontario, Canada), host and donor hearts were removed, and the coronary arteries were perfused with saline through the aorta and followed by light fixation by perfusion with $2 \%$ paraformaldehyde (Sigma Immunochemicals, St. Louis, MO). Because of previous descriptions indicating that cardiac allograft arteriopathy in the rabbit was equally distributed throughout the coronary circulation (34) and our own observations (28), the hearts were sectioned transversely from base to apex. Different sections of the hearts were either saved in $10 \%$ formalin (BDH Inc., Toronto, Ontario, Canada) for light microscopy studies or immediately frozen in O.T.C. Compound Tissue Tek (Miles Inc., Elkart, IN) for specific immunohistochemistry studies.

Grading of rejection. Tissue specimens from the donor hearts were stained with hematoxylin and eosin for histological grading of rejection according to a modified Billingham's criteria (35). The sections were graded by one of the senior pathologists (Dr. G. J. Wilson) at the Hospital For Sick Children without knowledge of whether the donor hearts came from control or CS1-treated animals.

\section{Quantitative assessment of host and donor coronary arteries by light microscopy}

Three different paraffin-embedded tissue sections from host and donor hearts from both control and CS1-treated rabbits were stained by the Movat pentachrome method for light microscopy. Morphometric analysis was performed using a Zeiss microscope attached to a computergenerated video analysis system (NuVision Software, Perceptics Inc., Knoxville, TN), as described previously (28). The number of vessels with intimal lesions was counted in all three heart sections from each experimental animal and was expressed as a percentage of total vessel number. In the host hearts, 999 vessels in the control group and 1,054 vessels in the CS1-treated group were analyzed. In the donor hearts, 827 vessels in the control group and 617 vessels in the CS1-treated group were analyzed. To determine the severity of intimal thickening, the diameter of each traceable vessel in all three sections was measured, and the coronary arteries were categorized as small (diameter $<100$ 
$\mu \mathrm{m}$ ), medium (diameter $>100<500 \mu \mathrm{m}$ ), and large (diameter $>500$ $\mu \mathrm{m})$. The degree of intimal thickening was then quantitatively assessed in each vessel size category as previously described (36). The areas encompassed by the outer medial layer (ML), the internal elastic lamina (IEL), and lumen were measured in each affected vessel, and the area of intimal thickening (IT) related to the vessel area was calculated by the formula IT $=$ IEL-lumen area/ML-lumen area $\times 100$.

\section{Immunohistochemistry studies}

In all immunohistochemistry analyses, we compared coronary arteries from host and donor hearts, with and without intimal thickening in the different size ranges from control and CS1-treated groups. The relative abundance of each specific antigen studied in the sections examined was graded semiquantitatively as minimal $(+/-)$, little $(+)$, moderately abundant $(++)$ to very abundant $(+++)$ by two investigators in our laboratory. The final scoring was based on individual gradings which reached $90 \%$ agreement.

Characterization of inflammatory cells. To characterize the presence of an immune-inflammatory reaction in the allograft coronary arteries in both groups studied, we performed immunoperoxidase staining using monoclonal antibodies to rabbit MHC class II antigens and rabbit $\mathrm{T}$ cells (a kind gift from Dr. Peter Libby, Brigham and Women's Hospital, Boston, MA), and also to rabbit macrophages (RAM 11; Dako Corp., Carpinteria, CA). The sections were air dried for $2 \mathrm{~h}$, fixed in acetone for $20 \mathrm{~min}$, and rinsed with Dulbecco (D)-PBS (Gibco, Burlington, Ontario, Canada)/0.1\% BSA (Boehringer-Mannheim, Mannheim, Germany). Endogenous peroxidase activity was blocked by immersing the sections in D-PBS $/ 0.1 \%$ BSA + 3\% hydrogen peroxide (BDH) for 30 min. After a nonspecific blocking step using $10 \%$ normal goat serum (Sigma Immunochemicals), the antibodies were applied to the sections for $1 \mathrm{~h}$ at a 1:10 dilution at room temperature. The sections were then rinsed, incubated with goat anti-mouse peroxidase-conjugated secondary antibody (Bio-Rad Laboratories, Richmond, CA) at a 1:50 dilution at room temperature for $45 \mathrm{~min}$, and developed with 3,3'-diaminobenzidine (DAB) (Sigma Immunochemicals) for $10 \mathrm{~min}$. Control sections were treated with normal mouse isotypic IgG (Dako Corp.).

Immune-detection of cellular adhesion molecules. To assess the influence of CS1 treatment on the expression of adhesion molecules in allograft coronary arteries, we performed immunoperoxidase staining for ICAM-1 and VCAM-1 on frozen sections of both host and donor hearts from the control and the CS1-treated groups. Monoclonal antibodies for ICAM-1 (mAb Rb2/3) and to VCAM-1 (mAb Rb1/9) were kindly supplied by Dr. Myron Cybulsky (Brigham and Women's Hospital ) and were used at a concentration of 1:10 for $1 \mathrm{~h}$ at room temperature. The procedure for immunostaining was essentially the same as described above.

Assessment of fibronectin. Fibronectin expression in coronary arteries of host and donor hearts from both control and CS1-treated groups was determined by performing immunoperoxidase staining using frozen sections. A monoclonal antibody anti-cellular fibronectin (Chemicon International, Inc., Temecula, CA) was used at a dilution of 1:100 for $1 \mathrm{~h}$ at room temperature, and the remaining details of the immunohistochemical procedure were essentially the same as described above. This antibody does not recognize plasma fibronectin.

\section{Statistical analysis}

The data are expressed as mean \pm SEM in Results and in the figure legends. In analyses related to the incidence and severity of lesions from both control and CS1-treated groups, the Student's $t$ test was used to test significance. The correlation among categorical variables from the immunohistochemistry studies, considered positive if $>+$ in the two groups (control and CS1-treated), was analyzed using Fisher's exact test. Differences were considered significant if $P<0.05$.

\section{Results}

Morphometric analyses of host and donor coronary arteries. Using light microscopic morphometric analysis applied to Mo-

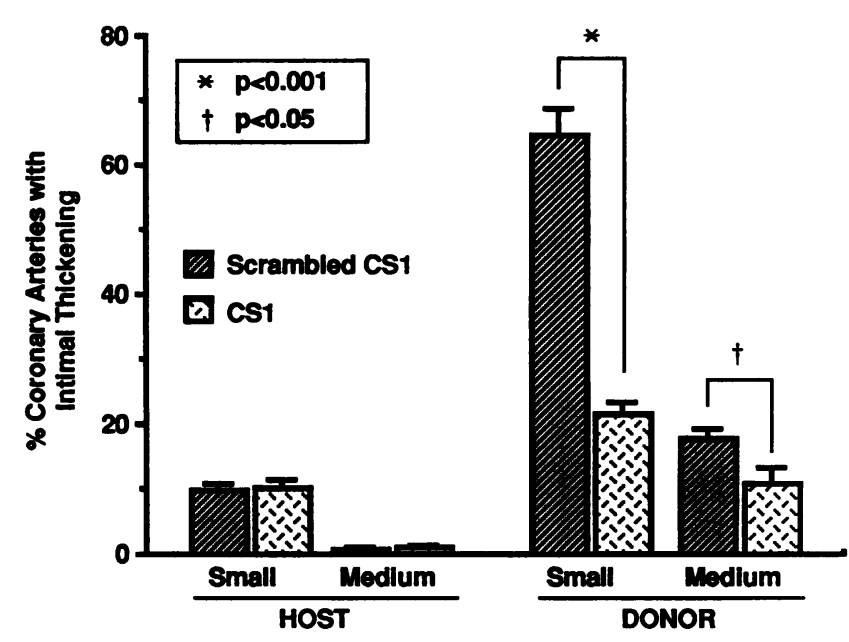

Figure 1. Effect of CS1 peptide treatment on the number of coronary arteries with intimal lesions in both host and donor hearts. The vast majority of vessels with intimal thickening were seen in small (diameter $\leq 100 \mathrm{~mm}$ ) and medium (diameter $>100 \leq 500 \mu \mathrm{m}$ ) size coronary arteries. The number of affected vessels in the CS1-treated group was significantly reduced compared with the control (scrambled CS1) group $(P<0.001$ for small size vessels and $P<0.05$ for medium size vessels), where a total of 617 vessels and 827 vessels, respectively, were analyzed. In the host coronary arteries, no differences were seen in both groups for small and medium size vessels, where a total of 1,054 vessels in the CS1-treated group and a total of 999 vessels in the control group were analyzed.

vat pentachrome-stained histologic sections, we observed a similar small proportion of coronary arteries with intimal thickening in host hearts from both control (scrambled peptide) and CS1-treated groups ( $10 \pm 1$ and $12 \pm 2 \%$ SEM, respectively) (Fig. 1). In donor hearts from the control group, however, $87 \pm 4 \%$ SEM of the vessels had intimal thickening, whereas in the CS1-treated group only $35 \pm 2 \%$ SEM were affected ( $P$ $<0.001$ for small size vessels and $P<0.05$ for medium size vessels) (Fig. 1). The proportion of coronary arteries with intimal thickening, in CS1 and control groups, was similarly distributed in the small ( $\leq 100 \mu \mathrm{m}$ diameter) and medium ( $>100$ $\leq 500 \mu \mathrm{m}$ diameter) size ranges; however, the large coronary arteries ( $>500 \mu \mathrm{m}$ diameter) were mostly spared.

The severity of the lesions, judged by the area of intimal thickening as a proportion of total vessel area, was similar in host coronary arteries from both control and CS1-treated groups (12 1 and $12 \pm 1 \%$ SEM, respectively) (Fig. 2). Intimal thickening in donor coronary arteries from control animals was three times more severe than that in host vessels, i.e., $36 \pm 2 \%$ SEM of total vessel area. In the CS1-treated group, intimal thickening was only $16 \pm 2 \%$ SEM $(P<0.001)$ (Fig. 2$)$. A similar reduction in the severity of intimal lesions in the CS1-treated compared with the control group was observed in both small ( $\leq 100$ $\mu \mathrm{m}$ diameter) and medium ( $>100 \leq 500 \mu \mathrm{m}$ diameter) size vessels. Representative examples of coronary arteries in the host, donor control, and donor CS1-treated animals are shown in Fig. 3, $A-C$, respectively. In addition, Fig. $4 A$ is representative of extensive intimal thickening affecting small vessels in the control group which contrasts with Fig. $4 B$, representing a markedly attenuated intimal lesion in small vessels in the CS1-treated group.

In contrast to the normal appearing myocardium in host 


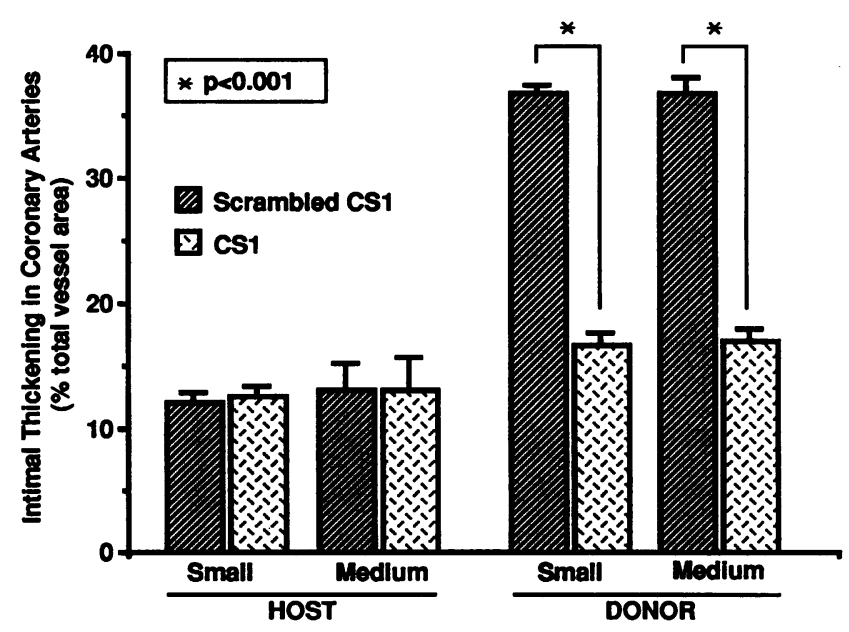

Figure 2. Effect of CS1 peptide treatment on the severity of coronary artery intimal lesions as assessed by intimal thickening (as percentage of total vessel area) related to vessel size in both host and donor hearts. There was significant reduction in the severity of intimal thickening in both small (diameter $\leq 100 \mathrm{~mm}$ ) and medium (diameter $>100 \leq 500$ $\mu \mathrm{m})$ size vessels of CS1-treated animals, compared with the control (scrambled CS1) group $(P<0.001)$, and that approached host levels. No differences in both groups were seen in host vessels, where the severity of intimal lesions was similarly low.

hearts (Fig. $3 \mathrm{D}$ ), myocardial rejection was of equal severity in donor control and donor CS1-treated hearts as judged by extensive lymphocytic infiltration and myocyte necrosis, hemorrhage, and fibrosis (Fig. 3, $E$ and $F$, respectively).

Immune-inflammatory markers in the coronary arteries. Immunohistochemical studies were performed to compare expres- sion of MHC class II molecules, T cells, and macrophages in host and donor coronary arteries from control and CS1-treated groups. Host coronary arteries were negative for these inflammatory markers. Fig. 5, $A$ and $D$, shows examples of negative immunostaining for MHC II and T cells, respectively. In donor hearts, however, there was enhanced expression of these markers of inflammation, albeit differing markedly in intensity in both control and CS1-treated animals. In five out of seven control animals, increased expression for MHC II molecules $(++$ to +++ ) was observed in donor coronary arteries (Fig. $5 B$ ), whereas in the CS1-treated group immunostaining in only two out of seven animals was abundant $(++)$, and was minimal $(+, \pm)$ or negative $(-)$ in the remainder (Fig. $5 C$ ) (Table I). While the difference in MHC expression was not reflected in statistical significance, we were able to show that CS1 treatment significantly decreased the presence of $\mathrm{T}$ cells in the coronary arteries (Fig. $5 F$ ). While five out of seven animals in the control group showed positive immunostaining of + \pm to ++ \pm , only one of seven CS1-treated animals showed $>+(+ \pm)$ expression (Fig. $5 E)(P<0.05)$ (Table I). Of note is the observation that the infrequent $\mathrm{T}$ cells observed in the CS1-treated group appeared to be mostly on the luminal surface (Fig. $5 F$ ) and also in the adventitia (Fig. $5 H$ ) with few cells seen infiltrating the vessel wall. On the other hand, the control group showed an increased proportion of $\mathrm{T}$ cells infiltrating the vessel wall (Fig. $5 E$, arrow), as well as present on the luminal surface (Fig. $5 E$ ) and adventitia (Fig. $5 G$ ). Macrophages were seldom observed in the host coronary arteries, and their presence in donor coronary arteries of both groups was also low, with no appreciable differences observed (Table I). However, macrophages were abundant around veins, at sites of intense myocardial infiltration of other inflammatory cells such as $\mathrm{T}$ cells, and this was associated with a similar degree of rejection in both CS1-treated and control groups.
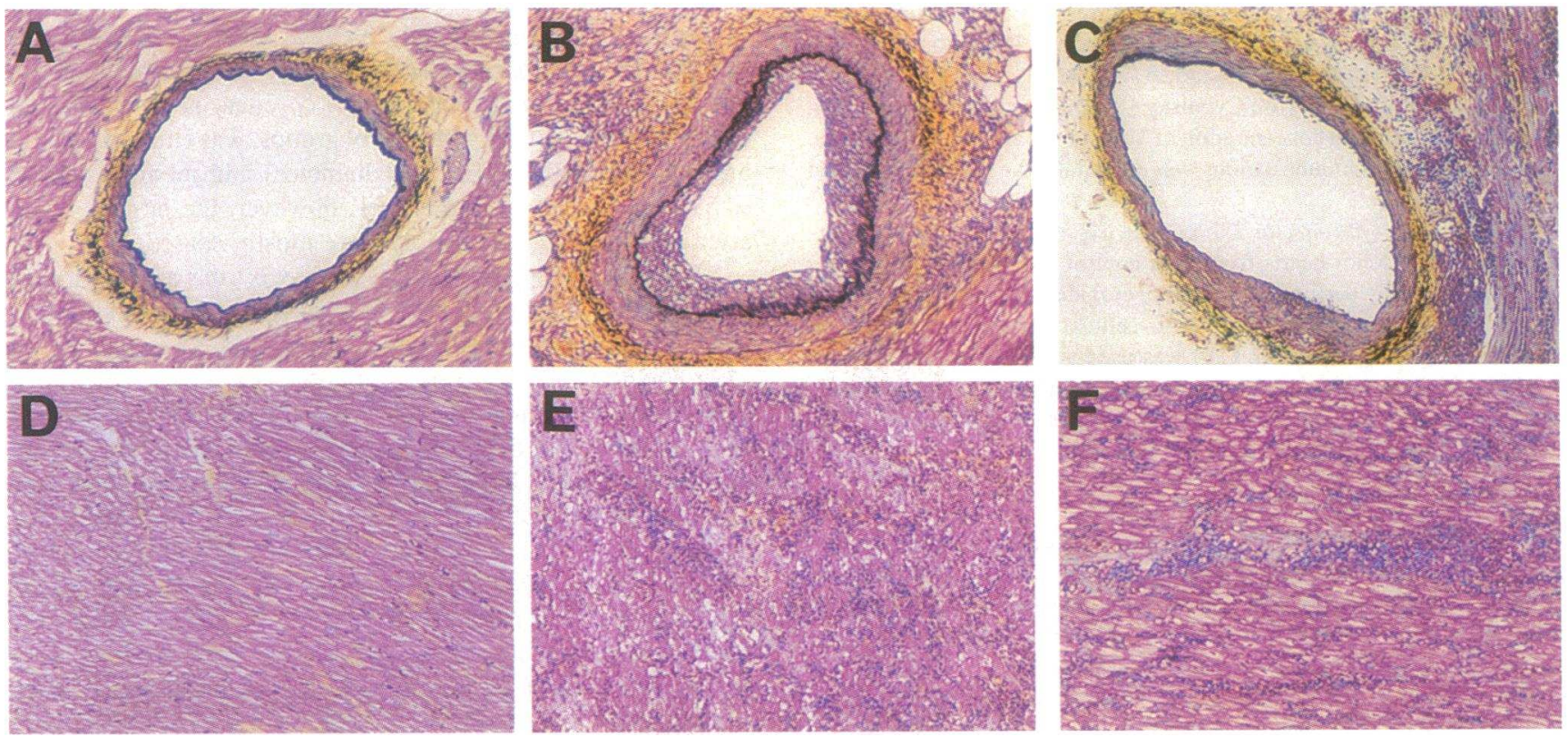

Figure 3. Representative photomicrographs of Movat pentachrome staining of coronary arteries in the host, donor control (scrambled CS1), and donor CS1-treated groups. The normal-appearing host vessel $(A)$ contrasts with the affected vessel showing a concentric intimal lesion $(B)$ in the control (scrambled CS1) group and a more normal appearing artery in the CS1-treated group $(C)$. Normal-appearing myocardium could be appreciated in host hearts $(D)$, which contrasts with severely rejected myocardium observed in both control $(E)$ and CS1-treated groups $(F)$. Note the presence of inflammatory cells. Original magnifications of $40(A-C)$ and $10(D-F)$. 

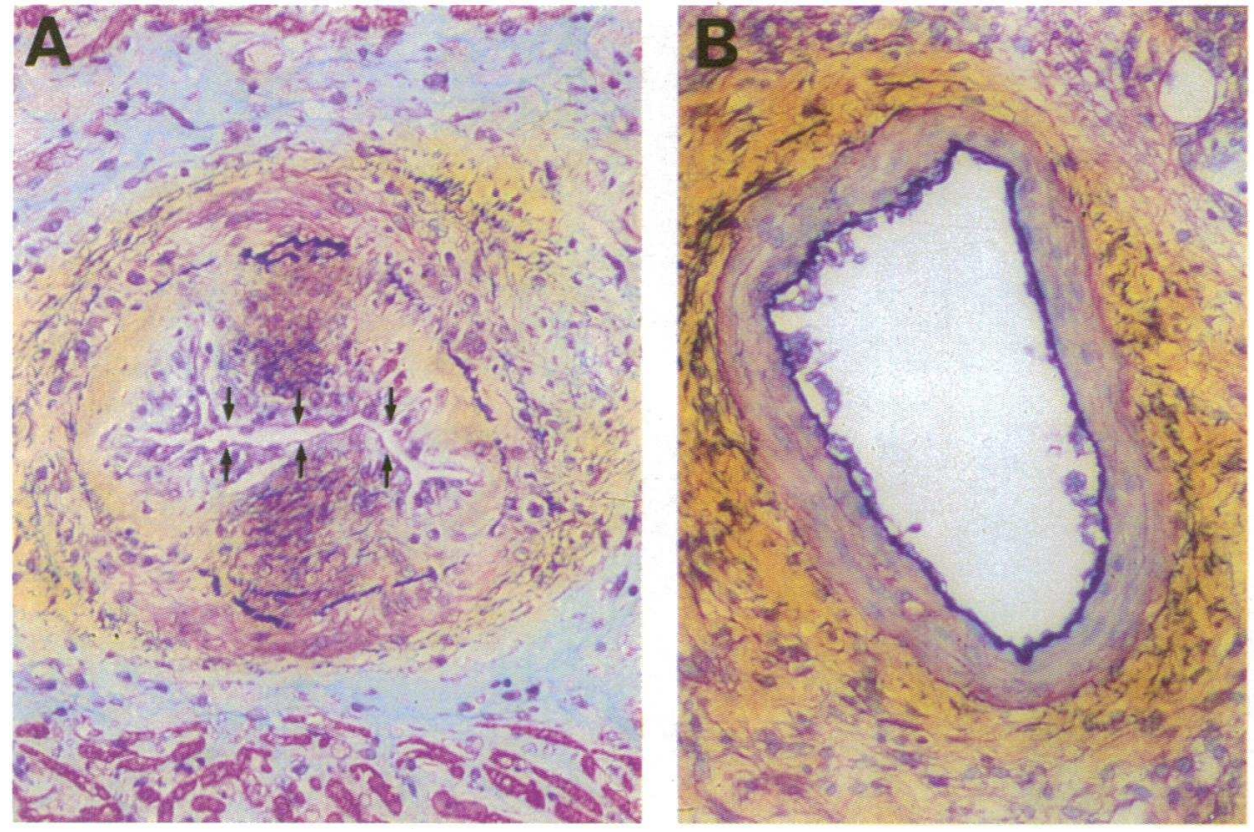

Figure 4. Representative photomicrographs of Movat pentachrome staining of small coronary arteries in donor control (scrambled CS1) and donor CS1-treated groups. Control animals had extensive intimal thickening in allograft small coronary arteries $(A$, arrows pointing to severe luminal occlusion), which contrasts with a markedly attenuated intimal lesion observed in allograft small coronary arteries from CS1-treated animals $(B)$.

Expression of cell adhesion molecules in the coronary arteries. The expression of both ICAM-1 and VCAM-1 was largely negative in the host hearts of both control and CS1-treated groups (see Fig. 6, $A$ and $D$, for respective examples). In the control donor coronary arteries, immunostaining for ICAM-1 was more prominent, with four out of seven animals graded $>+(+ \pm$ to ++$)($ Fig. $6 B)$, whereas in the CS1-treated group, ICAM-1 expression on the endothelial surface of coronary arteries was minimal with no animal showing an intensity $>+$ (Fig. $6 C)$. The difference in ICAM-1 immunostaining in the control and CS1-treated group trended toward significance $(P<0.06)$ (Table I). Likewise, VCAM-1 expression was increased in the donor coronary arteries of the control group (Fig. $6 E$ ), with four out of seven animals showing $>+$ intensity of immunostaining $(+ \pm$ to ++$)$, compared with none out of seven in the CS1-treated group, where VCAM-1 expression was minimal or absent (Fig. $6 F$ ) (Table I). This difference also represented a trend toward statistical significance $(P<0.06)$. In both control and CS1 groups, negative or minimal expression of adhesion molecules correlated with low levels of expression of MHC II and few T cells (Table I). There was, however, intense expression of ICAM-1 and VCAM-1 at venular sites, similar in both groups (data not shown). This was associated with infiltration of $\mathrm{T}$ cells and macrophages related to the severe myocardial rejection observed in allograft hearts.

Assessment of fibronectin accumulation in the coronary arteries. Minimal immunostaining for fibronectin was observed in host coronary arteries (Fig. 7, $A$ and $D$ ). In control donor coronary arteries, however, accumulation of fibronectin was abundant $(++$ to ++ \pm$)$ in six out of seven animals (Fig. 7, $B$ and $E$ ), whereas in the CS1-treated group minimal accumulation of fibronectin was observed ( - to \pm ) (Fig. 7, $C$ and $F$ ), with only one animal showing + intensity of immunostaining (Table I) $(P<0.003)$. The one animal negative for fibronectin in the control group showed minimal or negative expression of all other immune-inflammatory markers assessed in this study, and these findings correlated with the least number of vessels with intimal thickening and with reduced severity of the lesions in the control group (Table I).

\section{Discussion}

In this study, we describe the positive effect of a synthetic tetrapeptide, a short form of the CS1 peptide, in interfering with the development of experimental graft arteriopathy in vivo by specifically blocking the interaction between the $\alpha 4 \beta 1$ integrin receptor with the cell-associated matrix protein fibronectin. This peptide may also interfere with the transendothelial lymphocyte migration that is dependent on the interaction with the VCAM1 receptor on endothelial cell surfaces, albeit at much higher doses than those effective in blocking binding to fibronectin $(37,38)$. We were able to show a decrease in both the incidence and severity of allograft coronary artery lesions in the CS1treated compared with the control group, despite the fact that severe myocardial rejection was similar in both groups. Moreover, we observed a significant reduction in the infiltration of $\mathrm{T}$ cells in coronary arteries associated with a marked decrease in subendothelial fibronectin accumulation. Trends toward reduced expression of cell adhesion molecules (ICAM-1 and VCAM1) were also observed. These results indicate that blocking the initial interaction between fibronectin and $T$ cells alleviates the subsequent cytokine-mediated upregulation of fibronectin which we have shown contributes to the intimal thickening $(26,28)$. In addition, CS1 may directly block vascular smooth muscle fibronectin interaction and interfere with their migration into the subendothelium (30). This novel strategy which targets integrin receptors that are upregulated on the surface of immune-reactive cells, and expressed on vascular smooth muscle cells $(39,40)$, by blocking their interaction with cellular fibronectin, suggests an adjuvant therapeutic approach which may be useful in preventing or reducing the severity of graft arteriopathy.

The rabbit cardiac allograft model has been useful in studying a variety of pathophysiologic mechanisms either related to 

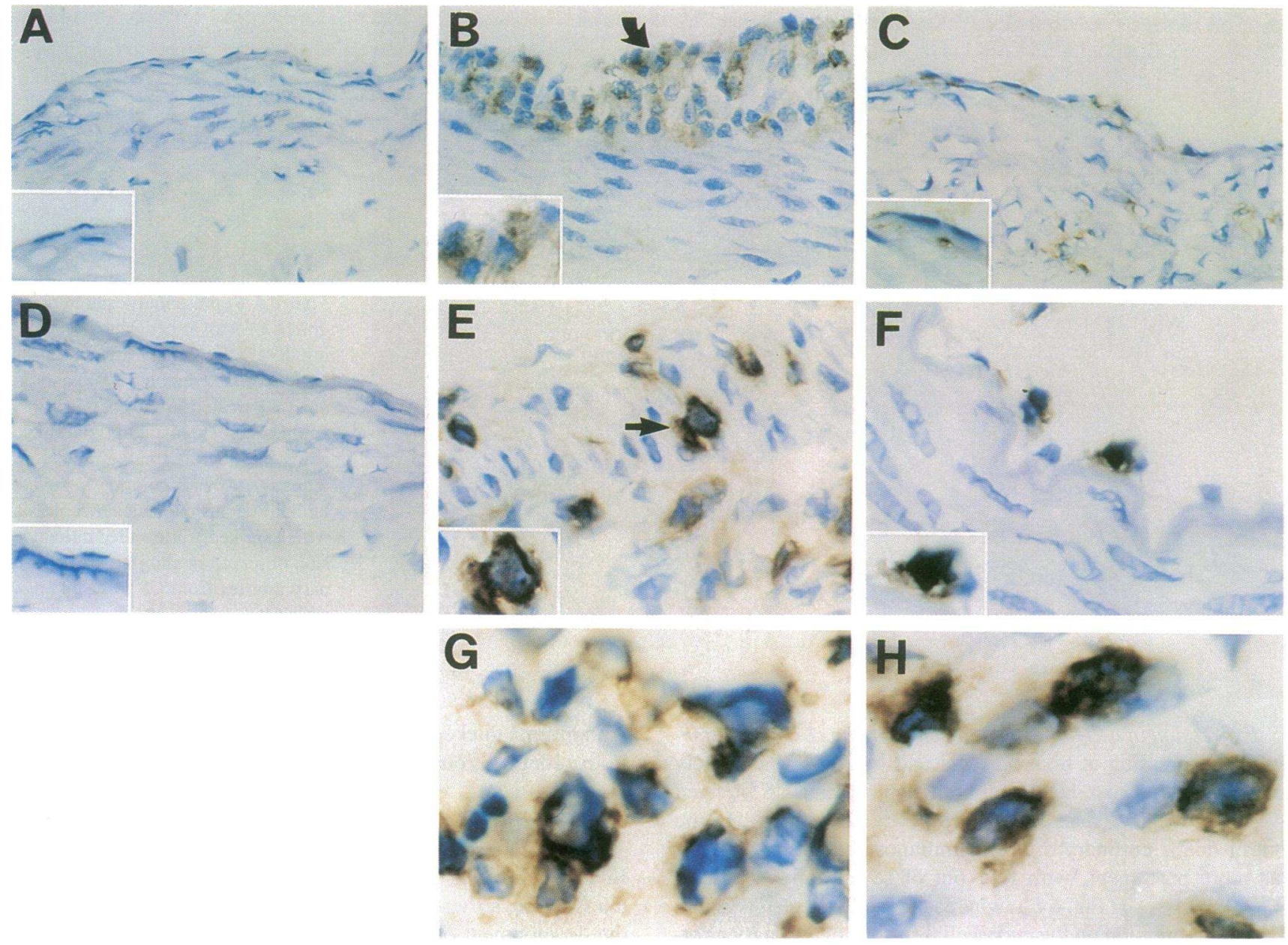

Figure 5. Representative photomicrographs of immunoperoxidase staining for MHC II $(A-C)$ and T cells $(D-H)$ in host and donor coronary arteries from both control (scrambled CS1) and CS1-treated groups. Negative staining was seen in most host vessels for both MHC II ( $A$ ) and T cells $(D)$. The expression of MHC II was more intense in some of the control animals, especially where intimal thickening was pronounced $(B$, arrow), compared with the CS1-treated group $(C)$. In the control group, $\mathrm{T}$ cells were abundant both on the endothelial surface and infiltrating the vessel wall $(E$, arrow). The presence of $\mathrm{T}$ cells was significantly reduced in the CS1 group, and when seen on the coronary arteries they were mostly adherent to the endothelial surface $(F)$. T cells could also be seen in the adventitia of coronary arteries in both CS1-treated $(H)$ and control groups $(G)$. Original magnifications of $40(A-F$; insets at an original magnification of 100$)$ and $100(G$ and $H)$.

the process of myocardial rejection or to the development of graft arteriopathy $(31,41)$. Despite the accelerated time course of the arteriopathy in this model $(28,31)$, the rabbit allograft arterial lesions resemble those observed in human allografts (41) and, therefore, the model offers the advantage of investigating, within a relatively short period of time, the pathophysiologic factors likely to be involved. It is the lack of immunosuppression that results in a shorter experimental time frame of 7$8 \mathrm{~d}$ as formerly described in this model $(28,31)$, since our previous studies have shown that neointimal thickening is not prominent in animals that are immunosuppressed (28).

A hypercholesterolemic diet was also introduced in the rabbit cardiac allograft model to further hasten the development of the arteriopathy (31), since rabbits have low systemic cholesterol levels (42), and also to more closely simulate the clinical setting, since hyperlipidemia is one of the risk factors accelerating the appearance of intimal lesion in human cardiac allografts $(1,43)$. The effect of a $0.5 \%$ cholesterol diet in the rabbit is reflected in increased circulating lipid levels (31), as well as the induction of early intimal lesions in host vessels, as judged by an average of $10-12 \%$ of vessels affected and a similar degree of vessel area with intimal thickening (12\%). Furthermore, we have observed fatty infiltration of the myocardium in rabbit allografts subjected to this diet (28).

The upregulation of integrin receptors, i.e., VLA-2, VLA4 , and VLA-6, has been shown in lung and heart biopsies of transplant patients undergoing episodes of rejection $(44,45)$. In addition, increased expression of matrix proteins bearing in their structure ligands for some of these integrins, i.e., fibronectin and laminin, was reported in rejected cardiac (46) and renal (47) allografts. These studies suggest that matrix could be involved in the recruitment of immune-reactive cells. Since the process of inflammatory cell emigration into tissues involves expression of adhesion molecules, e.g., ICAM-1, VCAM-1, and $P$ - and E-selectins on the endothelium $(48,49)$, there is increasing evidence that matrix proteins might further contribute by encouraging transendothelial migration and positioning.

In our study, we investigated the potential role of the interaction between the VLA-4 integrin and the CS1 motif in the fibronectin molecule in modulating inflammatory cell traffick- 
Table I. Morphometric and Immunohistochemical Findings in Allograft Coronary Arteries from Individual Cholesterol-fed Rabbits

\begin{tabular}{|c|c|c|c|c|c|c|c|c|c|c|}
\hline \multirow[b]{2}{*}{ Animal } & \multirow[b]{2}{*}{ Treatment } & \multirow{2}{*}{$\begin{array}{l}\text { Myocardial } \\
\text { rejection } \\
\text { grade }\end{array}$} & \multicolumn{8}{|c|}{ Coronary arteries } \\
\hline & & & MHC II & $\mathrm{T}$ cells & Macrophage & ICAM-1 & VCAM-1 & FN & $\begin{array}{l}\text { Number of vessels } \\
\text { with IT (\% total) }\end{array}$ & $\begin{array}{l}\text { Severity of IT } \\
\text { (\% vessel area) }\end{array}$ \\
\hline 1 & CS1 & 3 & \pm & + & \pm & + & \pm & \pm & $33 \%$ & $20 \%$ \\
\hline 2 & CS1 & 3 & ++ & + & + & + & + & + & $32 \%$ & $14 \%$ \\
\hline 3 & CS1 & 3 & ++ & + \pm & + & + & \pm & \pm & $47 \%$ & $21 \%$ \\
\hline 4 & CS1 & 3 & \pm & \pm & - & \pm & - & - & $32 \%$ & $11 \%$ \\
\hline 5 & $\mathrm{CS} 1$ & 3 & - & \pm & - & \pm & - & - & $36 \%$ & $20 \%$ \\
\hline 6 & $\mathrm{CS} 1$ & 3 & \pm & \pm & \pm & \pm & + & - & $25 \%$ & $11 \%$ \\
\hline 7 & CS1 & 3 & + & \pm & - & \pm & \pm & \pm & $41 \%$ & $18 \%$ \\
\hline 8 & CTRL & 3 & +++ & ++ \pm & + \pm & + \pm & ++ & ++ \pm & $95 \%$ & $40 \%$ \\
\hline 9 & CTRL & 3 & \pm & \pm & \pm & - & - & - & $67 \%$ & $24 \%$ \\
\hline 10 & CTRL & 3 & ++ & ++ & + & ++ & ++ & ++ \pm & $95 \%$ & $38 \%$ \\
\hline 11 & CTRL & 3 & ++ & + \pm & \pm & \pm & + & ++ & $75 \%$ & $33 \%$ \\
\hline 12 & CTRL & 3 & ++ & + \pm & \pm & ++ & + \pm & ++ & $89 \%$ & $37 \%$ \\
\hline 13 & CTRL & 3 & ++ & ++ & + \pm & + \pm & ++ & ++ & $98 \%$ & $40 \%$ \\
\hline 14 & CTRL & 3 & \pm & + & \pm & + & + & ++ & $94 \%$ & $39 \%$ \\
\hline
\end{tabular}

FN, fibronectin; IT, intimal thickening; CS1, CS1 peptide; CTRL, control (scrambled CS1 peptide);,,,,- \pm++++++ , negative, minimal, little, moderately abundant, and very abundant, respectively.

ing and in the development of the experimentally induced graft coronary arteriopathy. A synthetic CS1 tetrapeptide derived from the 25-mer sequence of the alternatively spliced CS1 motif in the fibronectin molecule, markedly decreased the number and reduced the severity of coronary artery intimal lesions in treated rabbits compared with a control group that received a scrambled form of the tetrapeptide CS1. Treatment with CS1 peptide did not appear to influence the number of host vessels with lesions or their severity, which were similarly low in the two groups studied. These latter findings differ from previous reports using other drugs, i.e., dehydroepiandrosterone (36) and angiopeptin (50), to abrogate graft arteriopathy in that those agents also reduced changes in host vessels and this might indicate a more selective effect related to the pathophysiology of the graft arteriopathy. The lack of host-related effect may, however, reflect the shorter time frame over which we assessed the development
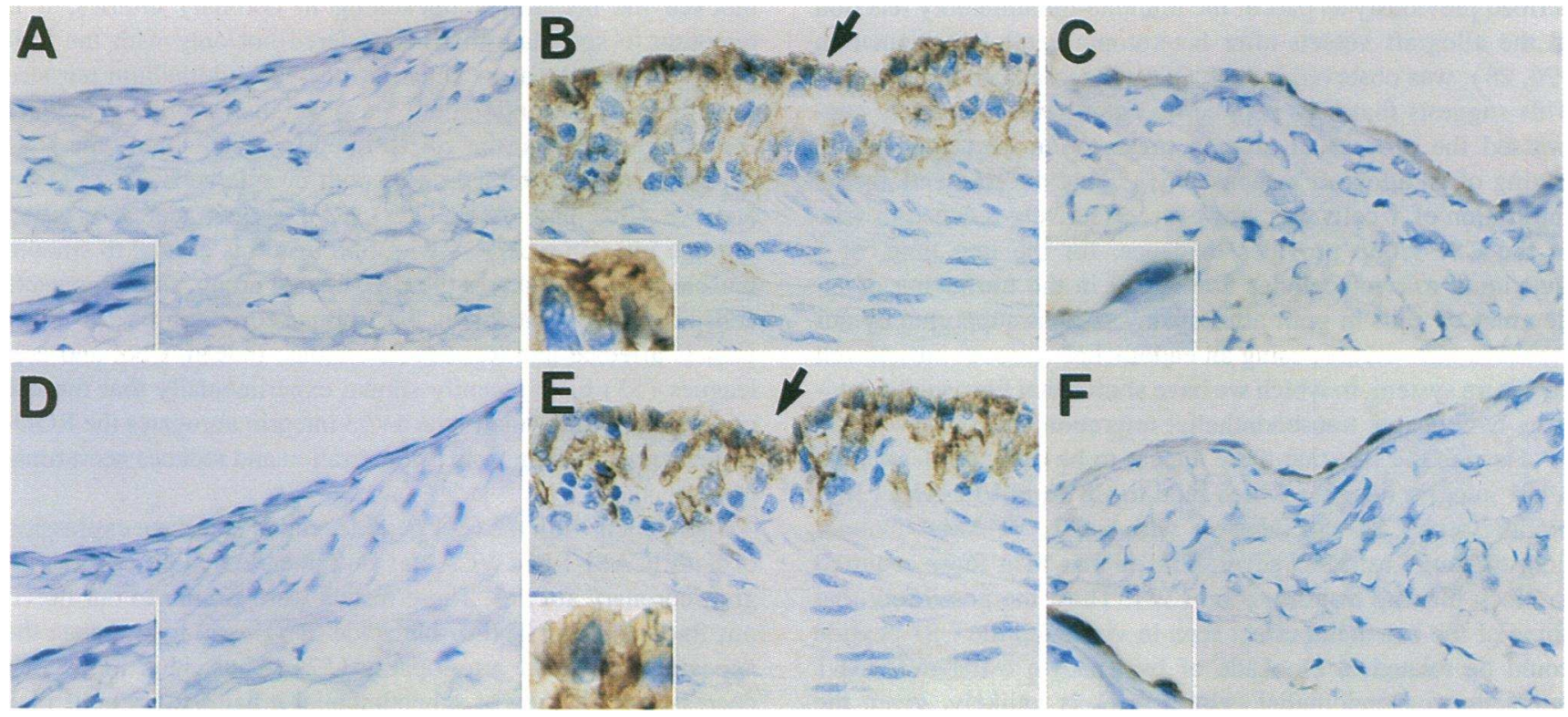

Figure 6. Representative photomicrographs of immunoperoxidase staining for ICAM-1 $(A-C)$ and VCAM-1 $(D-F)$ in host and donor coronary arteries of both control (scrambled CS1) and CS1-treated groups. Host coronary arteries were mostly negative for the expression of ICAM-1 and VCAM-1 ( $A$ and $D$, respectively). In the control group, there was increased expression of both ICAM-1 and VCAM-1 associated with endothelial cells but also with intimal cells where intimal thickening was observed (arrows in $B$ and $E$, respectively). There was marked reduction on the expression of both ICAM-1 and VCAM-1 in the CS1-treated group ( $C$ and $F$, respectively), where only some positive endothelial cells could be seen. Original magnification of 40; insets at an original magnification of 100. 

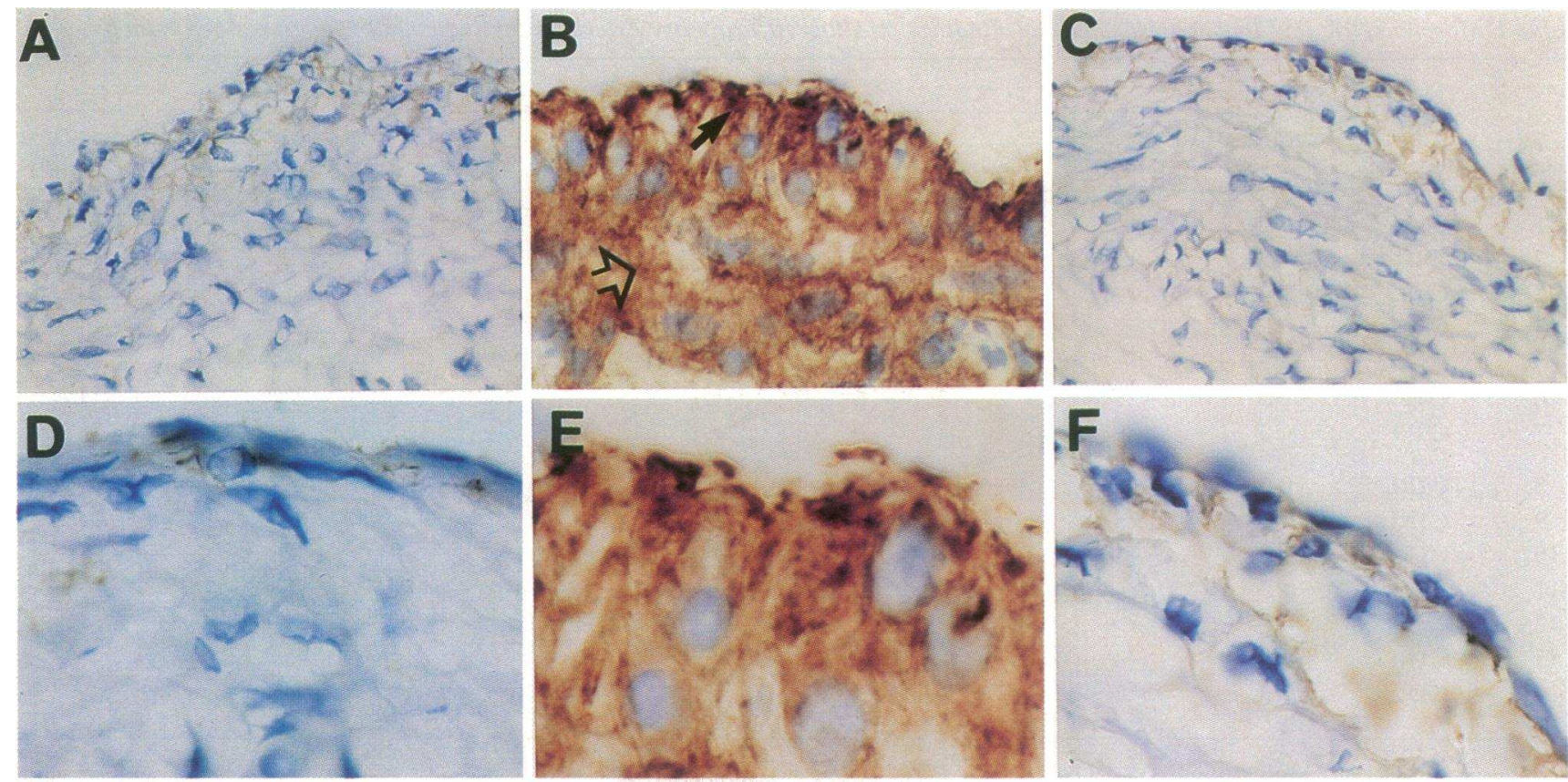

Figure 7. Representative photomicrographs of immunoperoxidase staining for cellular fibronectin in host and donor coronary arteries from both control (scrambled CS1) and CS1-treated groups. The accumulation of cellular fibronectin was minimal in host vessels, as seen under low and high magnifications ( $A$ and $D$, respectively). There was intense immunostaining in the control donor coronary arteries not only in the subendothelial space (closed arrow) but also throughout the medial layer (open arrow) $(B)$. Higher magnification is seen in $E$. In contrast, immunostaining for cellular fibronectin was reduced in the CS1-treated group $(C$ and $F$ ) and was of similar intensity to that seen in host vessels. $(A$ and $D)$. Original magnifications of $40(A-C)$ and $100(D-F)$.

of intimal lesions, i.e., $1 \mathrm{wk}$ without immunosuppressive therapy in this report versus 5-6 wk in the presence of immunosuppressive therapy in the aforementioned studies.

The expression of MHC class II molecules, which we described previously as part of the immune-inflammatory reaction in the allograft vessels after heterotopic heart transplantation $(26,28)$, was observed in both CS1-treated and control groups. This suggests that CS1 peptide may not have completely suppressed the process of antigen presentation occurring in the setting of an allograft response (51). That the transendothelial infiltration of $T$ cells was, however, effectively reduced in vivo in the CS1-group provides evidence, for the first time, of a functional role for cellular fibronectin in the trafficking of inflammatory cells in graft arteriopathy. This is supported by our recent in vitro studies using an endothelial-smooth muscle cell coculture system, in which we have shown that fibronectin regulates lymphocyte transendothelial migration (52).

Despite the fact that there appear to be distinct sites on the $\alpha 4 \beta 1$ integrin receptor which bind to CS1 and VCAM-1 (18), binding with CS1 can interfere with $\alpha 4 \beta 1-\mathrm{VCAM}-1$ interaction, although at doses severalfold higher than those required to block binding to fibronectin (37). Thus, the possibility that some of the beneficial effect seen in vivo with the CS1 peptide could be related to blockade of lymphocyte $\alpha 4 \beta 1$-VCAM-1 interaction on endothelial cell surfaces is unlikely, given the dose of compound used. Our in vitro data would suggest, however, that in this setting the effect of CS1 serves primarily to block interaction with fibronectin. That is, we have shown that CS1 and RGD peptides were equally effective and did not act synergistically in blocking transendothelial migration of lymphocytes in response to IL-1 $\beta$ stimulation of vascular smooth muscle cell fibronectin production (52). Had CS1 additionally blocked $\alpha 4 \beta 1$ interaction with VCAM-1, then one might have expected a greater inhibitory effect than with RGD alone.

On the other hand, given the efficacy with which CS1 blocked the neointimal thickening in coronary arteries, it is tempting to speculate that it interfered not only with the trafficking of inflammatory cells into the subendothelium but also with the migration of smooth muscle cells from the media into the intima. That is, the $\alpha 4 \beta 1$ integrin which binds the CS1 peptide is also expressed on smooth muscle cells $(17,39,40)$ and we (30) and others (53) have shown that interaction through integrin receptors with fibronectin is critical to smooth muscle cell migration. In the CS1-treated group, smooth muscle cells were less evident in the intima, correlating with fewer vessels affected and less severe lesions. Indeed, Choi and colleagues (53) have recently shown experimentally that the use of peptides which bind to the $\alpha \mathrm{v} \beta 3$ integrin abrogates the RGDdependent smooth muscle cell migration and reduces neointimal hyperplasia.

Treatment with the CS1 peptide tended to reduce expression of both ICAM-1 and VCAM-1 on the endothelium of the allograft coronary arteries. These results were similar to our previous findings using TNF- $\alpha$ blockade (TNF- $\alpha$ sr) to attenuate the appearance of graft arteriopathy (52). Thus, it is likely that decreased trafficking of subendothelial inflammatory cells may result in reduced expression of cytokines and less induction of adhesion molecules. A similar mechanism may explain the reduced fibronectin accumulation in the coronary arteries of CS1-treated rabbits. In this regard, we have reported previously that fibronectin is upregulated by increased endothelial and smooth muscle cell production of cytokines, i.e., IL- $1 \beta$ and 
TNF- $\alpha(3,4,27)$, and it is likely that release of these cytokines from inflammatory cells leads to their induction in vascular cells (2).

Macrophages were observed less frequently in the donor coronary arteries of both experimental groups, and this is in keeping with our previous in vivo studies in rabbits and piglets in which macrophages were not a prominent early feature of the accelerated graft arteriopathy. Kuwahara et al. (42) have reported the presence of macrophages in vascular lesions from rejected rabbit cardiac allografts at 2 and 3 wk after transplantation, with only lymphocytes evident after 1 wk. Lipid-laden macrophages are certainly evident in coronary arteries in patients that develop graft arteriopathy years after cardiac transplantation (54). Macrophages were also seen at venular sites among the clusters of inflammatory cells, including $\mathrm{T}$ cells, infiltrating the rejected myocardium in both CS1-treated and control groups, findings similar to those demonstrated in other studies (55). The expression of adhesion molecules was also intense at these venular sites. This would indicate that different qualitative or quantitative factors are responsible for myocardial rejection and graft arteriopathy. Thus, this supports our previous experience with the TNF- $\alpha$ sr which preferentially also blocked graft arteriopathy but not myocardial rejection, as well as clinical experience showing that graft arteriopathy occurs despite immunosuppressive therapy and absence of acute episodes of rejection (56).

In summary, we have used specific reagents to show that the reduction of an accelerated form of graft arteriopathy, induced in cholesterol-fed rabbits after cardiac transplantation, can be achieved by blockade of the interaction between VLA4 integrin and cell-associated fibronectin. We propose that this halts the subsequent cytokine-dependent cascade of events resulting from activated $T$ cell subendothelial trafficking and positioning. The latter involves upregulation of adhesion molecules and further induction of fibronectin expression. Treatment with CS1 specifically blocked vascular changes, since myocardial rejection was as severe in this group of animals as in the control group. Therefore, it is possible that targeting the interaction between fibronectin and VLA-4 on immune-inflammatory cells may offer adjuvant therapies effective in preventing graft arteriopathy.

\section{Acknowledgments}

We would like to thank Mike Starr for preparing the color photomicrographs and Joan Jowlabar and Susy Taylor for secretarial assistance. We are also indebted to the staff at the Animal Care Facility at The Hospital For Sick Children for helping with the animal care.

This work was supported by a grant from the Medical Research Council of Canada (PG12351).

\section{References}

1. Salomon, R., C. Hughes, F. Schoen, D. Payne, J. Pober, and P. Libby. 1991. Human coronary transplantation-associated arteriosclerosis. Am. J. Pathol. 138:791-798.

2. Libby, P., S. Swanson, H. Tanaka, A. Murray, F. Schoen, and J. Pober. 1992. Immunopathology of coronary arteriosclerosis in transplanted hearts. $J$. Heart Lung Transplant. 11:S5-S6.

3. Clausell, N., and M. Rabinovitch. 1993. Upregulation of fibronectin synthesis by interleukin- $1 \beta$ in coronary artery smooth muscle cells is associated with the development of the post-cardiac transplant arteriopathy in piglets. J. Clin. Invest. 92:1850-1858.

4. Molossi, S., N. Clausell, and M. Rabinovitch. 1993. Coronary artery endo- thelial interleukin- $1 \beta$ mediates enhanced fibronectin production related to postcardiac transplant arteriopathy in piglets. Circulation.. 88:248-256.

5. Carlos, T., B. Schwartz, N. Kovach, E. Yee, M. Rosa, L. Osborn, G. ChiRosso, B. Newman, R. Lobb, and J. Harlan. 1990. Vascular cell adhesion molecule-1 mediates lymphocyte adherence to cytokine-activated cultured human endothelial cells. Blood. 76:965-970.

6. Jonjic, N., P. Jilek, S. Bernasconi, G. Peri, I. Martin-Padura, S. Cenzuales, E. Dejana, and A. Mantovani. 1992. Molecules involved in the adhesion and cytotoxicity of activated monocytes on endothelial cells. J. Immunol .. 148:20802083.

7. de Sousa, M., N. Tilney, and J. Kupiec-Weglinski. 1991. Recognition of self within self: specific lymphocyte positioning and the extracellular matrix. Immunol.Today. 12:262-266.

8. Wayner, E., A. Garcia-Pardo, M. Humphries, J. McDonald, and W. Carter. 1989. Identification and characterization of the $T$ lymphocyte adhesion receptor for an alternative cell attachment domain (CS1) in plasma fibronectin. J. Cell Biol. 109:1321-1330.

9. Matsuyama, T., A. Yamada, J. Kay, K. Yamada, S. Akiyama, S. Schlossman, and C. Morimoto. 1989. Activation of CD4 cells by fibronectin and antiCD3 antibody. A synergistic effect mediated by the VLA-5 fibronectin receptor complex. J. Exp. Med. 170:1133-1148.

10. Hershkoviz, R., D. Gilat, S. Miron, Y. Mekori, D. Aderka, D. Wallach, I. Vlodavsky, I. Cohen, and O. Lider. 1993. Extracellular matrix induces tumor necrosis factor- $\alpha$ secretion by an interaction between resting rat $\mathrm{CD} 4^{+} \mathrm{T}$ cells and macrophages. Immunology. 78:50-57.

11. Shimizu, Y., W. Newman, Y. Tanaka, and S. Shaw. 1992. Lymphocyte interactions with endothelial cells. Immunol. Today. 13:106-112.

12. Li, Y., and H. Cheung. 1992. Basement membrane and its components on lymphocyte adhesion, migration, and proliferation. J. Immunol. 149:31743181.

13. van Seventer, G., Y. Shimizu, and S. Shaw. 1991. Roles of multiple accessory molecules in T-cell activation. Curr. Opin. Immunol.. 3:294-303.

14. Guan, J.-L., and R. O. Hynes. 1990. Lymphoid cells recognize an alternatively spliced segment of fibronectin via the integrin receptor $\alpha_{4} \beta_{1}$. Cell. 60:5361.

15. Shimizu, Y., G. van Seventer, K. Horgan, and S. Shaw. 1990. Roles of adhesion molecules in $\mathrm{T}$-cell recognition: fundamental similarities between four integrins on resting human T cells (LFA-1, VLA-4, VLA-5, VLA-6) in expression, binding and costimulation. Immunol. Rev. 114:109-142.

16. Garcia-Pardo, A., E. Wayner, W. Carter, and O. Ferreira, Jr. 1990. Human B lymphocytes define an alternative mechanism of adhesion to fibronectin. $J$. Immunol. 144:3361-3366.

17. Hynes, R. O. 1992. Integrins: versatility, modulation, and signaling in cell adhesion. Cell. 69:11-25.

18. Elices, M., L. Osborn, Y. Takada, C. Crouse, S. Luhowskyj, M. Hemler, and R. Lobb. 1990. VCAM-1 on activated endothelium interacts with the leukocyte integrin VLA-4 at a site distinct from the VLA-4/fibronectin binding site. Cell. 60:577-584.

19. Gismondi, A., S. Morrone, M. Humphries, M. Piccoli, L. Frati, and A. Santoni. 1991. Human natural killer cells express VLA-4 and VLA-5, which mediate their adhesion to fibronectin. J. Immunol. 146:384-392.

20. Chuluyan, H., and A. Issekutz. 1993. VLA-4 integrin can mediate CD11/ CD18-independent transendothelial migration of human monocytes. J. Clin. Invest. 92:2768-2777.

21. Anwar, A., R. Moqbel, G. Walsh, A. Kay, and A. Wardlaw. 1993. Adhesion to fibronectin prolongs eosinophil survival. J. Exp. Med. 177:839-843.

22. Takeuchi, T., K. Amano, H. Sekine, J. Koide, and T. Abe. 1993. Upregulated expression and function of integrin adhesive receptors in systemic lupus erythematosus patients with vasculitis. J. Clin. Invest. 92:3008-3016.

23. Laffon, A., R. Garcia-Vicuna, A. Humbria, A. A. Postigo, A. L. Corbi, M. O. de Landazuri, and F. Sanchez-Madrid. 1991. Upregulated expression and function of VLA-4 fibronectin receptors on human activated T cells in rheumatoid arthritis. J. Clin. Invest. 88:546-552.

24. Elices, M., V. Tsai, D. Strahl, A. Goel, V. Tollefson, T. Arrhenius, E. Wayner, F. Gaeta, J. Fikes, and G. Firestein. 1994. Expression and functional significance of alternatively spliced CS1 fibronectin in rheumatoid arthritis microvasculature. J. Clin. Invest. 93:405-416.

25. Ager, A., and M. Humphries. 1991. Use of synthetic peptides to probe lymphocyte-high endothelial cell interactions. Lymphocytes recognize a ligand on the endothelial surface which contains the CS1 adhesion motif. Int Immunol. 2:921-928.

26. Clausell, N., S. Molossi, and M. Rabinovitch. 1993. Increased interleukin$1 \beta$ and fibronectin expression are early features of the development of the postcardiac transplant coronary arteriopathy in piglets. Am. J. Pathol. 142:1772-1786.

27. Molossi, S., N. Clausell, and M. Rabinovitch. 1995. Reciprocal induction of tumor necrosis factor-a and interleukin-1b activity mediates fibronectin synthesis in coronary artery smooth muscle cells. J. Cell. Physiol. 163:19-29.

28. Clausell, N., S. Molossi, S. Sett, and M. Rabinovitch. 1994. In vivo blockade of tumor necrosis factor-a in cholesterol-fed rabbits following cardiac 
transplant inhibits acute coronary artery neointimal formation. Circulation. 89:2768-2779.

29. Molossi, S., N. Clausell, S. Sett, and M. Rabinovitch. 1994. Neutralization of tumor necrosis factor-alpha reduces expression of vascular adhesion molecules and associated intimal thickening in rabbit cardiac allografts. J. Am. Coll. Cardiol. Feb:140A

30. Boudreau, N., E. Turley, and M. Rabinovitch. 1991. Fibronectin, hyaluronan and a hyaluronan binding protein contribute to increased ductus arteriosus smooth muscle cell migration. Dev. Biol. 143:235-247.

31. Alonso, D., P. Starek, and C. Minick. 1977. Studies on the pathogenesis of athero-arteriosclerosis induced in rabbit cardiac allografts by the synergy of graft rejection and hypercholesterolemia. Am. J. Pathol. 87:415-442.

32. Komoriya, A., L. Green, M. Mervic, S. Yamada, K. Yamada, and M. Humphries. 1991. The minimal essential sequence for major cell type-specific adhesion site (CS1) within the alternatively spliced type III connecting segment domain of fibronectin is leucine-aspartic acid-valine. J. Biol. Chem. 266:1507515079.

33. Wayner, E. A., and N. L. Kovach. 1992. Activation-dependent recognition by hematopoietic cells of the LDV sequence in the $\mathrm{V}$ region of fibronectin. $J$. Cell Biol. 116:489-497.

34. Foegh, M., B. Khirabadi, E. Chambers, and P. Ramwell. 1989. Peptide inhibition of accelerated transplant atherosclerosis. Transplant. Proc. 21:36743676.

35. Billingham, M. 1979. Some recent advances in cardiac pathology. Hum. Pathol. 10:367-386.

36. Eich, D., J. Nestler, D. Johnson, G. Dworkin, D. Ko, A. Wechsler, and M. Hess. 1993. Inhibition of accelerated coronary atherosclerosis with dehydroepiandrosterone in the heterotopic rabbit model of cardiac transplantation. Circulation. 87:261-269.

37. Makarem, R., P. Newham, J. Askari, L. Green, J. Clements, M. Edwards, M. Humphries, and P. Mould. 1994. Competitive binding of vascular cell adhesion molecule-1 and the HepII/III domain of fibronectin to the integrin $a_{4} b_{1}$. J. Biol. Chem. 269:4005-4011.

38. Romanic, A., and J. Madri. 1994. The induction of 72-kD gelatinase in T cells upon adhesion to endothelial cells is VCAM-1 dependent. J. Cell Biol. 125:1165-1178.

39. Hynes, R. O. 1987. Integrins: a family of cell surface receptors. Cell. 48:549-554.

40. Ruoslahti, E. 1991. Integrins. J. Clin. Invest. 87:1-5.

41. Foegh, M. 1993. Accelerated cardiac transplant atherosclerosis/chronic rejection in rabbits: inhibition by angiopeptin. Transplant. Proc. 25:2095-2097.

42. Kuwahara, M., J. Jacobsson, M. Kuwahara, E. Kagan, P. Ramwell, and
M. Foegh. 1991. Coronary artery ultrastructural changes in cardiac transplant atherosclerosis in the rabbit. Transplantation (Baltimore). 52:759-765.

43. Johnson, M. 1992. Transplant coronary disease: nonimmunologic risk factors. J. Heart Lung Transplant. 11:S124-S132.

44. Steinhoff, G., M. Behrend, N. Richter, and A. Haverich. 1994. Distinct expression of cell-cell and cell-matrix adhesion molecules on endothelial cells in heart and lung transplants. J. Heart Lung Transplant. 13:S55a. (Abstr.)

45. Lemstrom, K. B., P. K. Koskinen, and P. J. Hayry. 1994. Expression of adhesion ligand molecules in rat cardiac allograft rejection. J. Heart Lung Transplant. 13:S64a. (Abstr.)

46. Coito, A. J., J. Binder, M. de Sousa, and W. J. W. Kupiec. 1994. The expression of extracellular matrix proteins during accelerated rejection of cardiac allografts in sensitized rats. Transplantation (Baltimore). 57:599-605.

47. Gould, V., V. Martinez-Lacabe, I. Virtanen, K. Sahlin, and M. Schwartz. 1992. Differential distribution of tenascin and cellular fibronectins in acute and chronic renal allograft rejection. Lab. Invest. 67:71-79.

48. Osborn, L. 1990. Leukocyte adhesion to endothelium in inflammation Cell. 62:3-6.

49. Smith, C., J. Barker, and T. Lee. 1993. Adhesion molecules in allergic inflammation. Am. Rev. Respir. Dis. 148(Suppl.):S75-S78.

50. Foegh, M., B. Khirabadi, E. Chambers, S. Amamoo, and P. Ramwell. 1989. Inhibition of coronary artery transplant atherosclerosis in rabbits with angiopeptin, an octapeptide. Atherosclerosis. 78:229-236.

51. Krensky, A., A. Weiss, G. Crabtree, M. Davis, and P. Parham. 1990. Tlymphocyte-antigen interactions in transplant rejection. N. Engl. J. Med. 322:510517.

52. Molossi S., M. Elices, T. Arrhenius, and M. Rabinovitch. 1995. Lymphocyte transendothelial migration toward smooth muscle cells in interleukin-1 $\beta$ stimulated co-cultures is related to fibronectin interactions with $\alpha_{4} \beta_{1}$ and $\alpha_{5} \beta_{1}$ integrins. J. Cell. Physiol. In press.

53. Choi, E. T., L. Engel, A. D. Callow, S. Sun, J. Trachtenberg, S. Santoro, and U. S. Ryan. 1994. Inhibition of neointimal hyperplasia by blocking $\alpha_{\mathrm{v}} \beta_{3}$ integrin with a small peptide antagonist GpenGRGDSPCA. J. Vasc. Surg. 19:125-134.

54. Billingham, M. 1992. Histopathology of graft coronary disease. J. Heart Lung Transplant. 11:S38-S44.

55. Gassel, A., M. Radzun, H. Hansmann, M. Weyand, and W. Konertz. 1989. Monocytes and macrophages in the rejection of human cardiac allografts. Transplant. Proc. 21:2514-2516.

56. Anderson, T., I. Meredith, A. Uehata, G. Mudge, A. Selwyn, P. Ganz, and A. Yeung. 1993. Functional significance of intimal thickening as detected by intravascular ultrasound early and late after cardiac transplantation. Circulation. 88:1093-1100. 